\title{
Directed percolation in nonunitary quantum cellular automata
}

\author{
Ramil Nigmatullin $\odot,{ }^{*}$ Elisabeth Wagner $\odot$, and Gavin K. Brennen $\odot$ \\ Center for Engineered Quantum Systems, Department of Physics \& Astronomy, Macquarie University, 2109 NSW, Australia
}

(Received 19 May 2021; accepted 3 November 2021; published 10 December 2021)

\begin{abstract}
Probabilistic cellular automata (CA) provide a classic framework for studying nonequilibrium statistical physics on lattices. A notable example is the Domany-Kinzel CA, which has been used to investigate the process of directed percolation and the critical dynamics of the nonequilibrium phase transition between absorbing and percolating phases. In this work, we construct a nonunitary quantum CA that generalizes the Domany-Kinzel CA and study the resulting dynamical evolution using numerical simulations using the tensor network infinite time-evolving block decimation (iTEBD) algorithm. We demonstrate that the system undergoes the absorbing/percolating phase transition and that the addition of the Hamiltonian generates coherences, which are a distinct feature of quantum dynamics. A proposal for the implementation of the model with Rydberg array is put forward, which does not require local addressing of individual sites.
\end{abstract}

DOI: 10.1103/PhysRevResearch.3.043167

\section{INTRODUCTION}

In recent years there have been great advances in the development of quantum simulation platforms. These include ultracold atoms, ions, superconducting qubits, and photonic systems. One of the most recent advances has been the development of a quantum simulator based on arrays of ultracold Rydberg atoms [1]. Atoms that are excited to Rydberg states interact strongly, which makes it possible to engineer controlled multiqubit dynamics. Rydberg arrays provide an excellent platform for studying nonequilibrium dynamics of quantum many-body systems, for example, for probing nonequilibrium quench dynamics in Ising spin chains [2,3], energy transport $[4,5]$, and the validity of thermalization hypothesis $[6,7]$.

In the domain of nonequilibrium physics, one promising application of Rydberg arrays is in the exploration of nonequilibrium phase transitions (NEPTs), the study of which is particularly challenging due to the requirement of large system sizes and the long-time evolution needed to reach steady states. The classical NEPTs are classified into universality classes characterized by power scaling laws at critical points $[8,9]$. Quantum fluctuations can drastically alter the nature of NEPT; for example, it was shown that the critical exponents of NEPT in a quantum analog of the contact process model [10] are different from the critical exponents of a classical model $[11,12]$. The quantum generalization of NEPT theory remains largely unexplored both theoretically and experimentally because of the challenges in simulating the dynamics of

\footnotetext{
*ramil.nigmatullin@mq.edu.au

Published by the American Physical Society under the terms of the Creative Commons Attribution 4.0 International license. Further distribution of this work must maintain attribution to the author(s) and the published article's title, journal citation, and DOI.
}

quantum many-body systems and engineering such systems in the laboratory.

Perhaps the most fundamental NEPT is the phase transition between the absorbing and percolating phases in lattice systems undergoing directed percolation (DP) dynamics [8]. One extensively studied microscopic model exhibiting DP dynamics is the Domany-Kinzel Cellular Automaton (DKCA). A quantum generalization of DKCA, which can be implemented using $2 \mathrm{~d}$ Rydberg arrays, has been recently proposed [13] and studied numerically [14-16]. The model in Ref. [13] involves application of local 3-site unitary gates, which sequentially updates the rows, such that the state of each row depends on the state of the previous row and corresponds to the state of a probabilistic CA undergoing DP dynamics.

In this paper, we construct a novel quantum DKCA model, which uses a combination of nontrivial dissipative dynamics as well as coherent nearest-neighbor dynamics. The scheme is inspired by the recently developed techniques for engineering dissipative interactions in Rydberg arrays [17]. The strength of the influence of quantum effects can be varied by tuning the relative contribution of coherent and stochastic terms to the overall dynamics. The proposed scheme has several advantages. In particular, it does not require addressing the individual lattice sites as all of the sites can be updated simultaneously with spatially homogeneous dynamics. The scheme can also be viewed as a robust way of preparing many-body quantum states, since the reached steady states are independent of the initially prepared state. The approach can be easily generalized to $(2+1)$-dimensional quantum $\mathrm{CA}$ for probing more complex nonequilibrium models.

The paper is organized as follows. Section II describes the construction of the nonunitary QCA rules that generate the DP process. Section III describes the simulation method, and Sec. IV presents the results and draws the comparison with the classical stochastic DP. Finally, the possible physical implementation in Rydberg arrays is discussed in Sec. V. 


\section{MODEL}

We consider a one-dimensional spin lattice, which undergoes open quantum dynamical evolution modeled using a Markovian master equation. Both the Hamiltonian and the Lindblad jump operator in the master equation are 3-qubit operators coupling the nearest-neighboring sites in the array. The master equation is given by

$$
\partial_{t} \rho=\mathcal{L}[\rho]=-i[H, \rho]+\mathcal{D}[\rho],
$$

where the Hamiltonian is of the form

$$
H=\frac{1}{2} \sum_{j} \sum_{\alpha \beta} \theta_{\alpha \beta}|\alpha\rangle\left\langle\left.\alpha\right|_{j-1} \otimes X_{j} \otimes \mid \beta\right\rangle\left\langle\left.\beta\right|_{j+1},\right.
$$

where $\alpha, \beta \in\{0,1\},|\alpha\rangle\left\langle\left.\alpha\right|_{j}\right.$ is a projector on site $j, X_{j}$ is the Pauli-X operator on site $j$, and $\theta_{\alpha \beta}$ are constants whose values can be experimentally tuned.

The dissipator is given by

$$
\begin{aligned}
\mathcal{D}[\rho]= & \sum_{j}\left[L_{j}^{+} \rho L_{j}^{+\dagger}-\frac{1}{2}\left(L_{j}^{+\dagger} L_{j}^{+} \rho+\rho L_{j}^{+\dagger} L_{j}^{+}\right)\right. \\
& \left.+L_{j}^{-} \rho L_{j}^{-\dagger}-\frac{1}{2}\left(L_{j}^{-\dagger} L_{j}^{-} \rho+\rho L_{j}^{-\dagger} L_{j}^{-}\right)\right],
\end{aligned}
$$

with the jump operators

$$
L_{j}^{ \pm}=\sum_{\alpha \beta} \sqrt{\gamma_{\alpha \beta}^{ \pm}}|\alpha\rangle\left\langle\left.\alpha\right|_{j-1} \otimes \sigma_{j}^{ \pm} \otimes \mid \beta\right\rangle\left\langle\left.\beta\right|_{j+1},\right.
$$

where $\sigma_{j}^{ \pm}=X_{j} \pm i Y_{j}$ is a lowering/raising operator on site $j$, and $\gamma_{\alpha \beta}^{ \pm}$are tunable rate constants. If the Hamiltonian term is set to zero, $\theta_{\alpha \beta}=0$ for all $\alpha, \beta \in\{0,1\}$, then the master equation describes classical stochastic dynamics on a lattice since no coherence can be generated if the system is initialized in a product state. Such dynamics can be realized in atomic systems such as trapped ions and Rydberg atom arrays. A possible implementation in a Rydberg array will be described in Sec. V.

We will now address the question of how to appropriately choose the rates $\gamma_{\alpha \beta}^{ \pm}$, such that the model would correspond to the process of DP in the fully stochastic incoherent regime. The idea is to choose the Lindblad operators such that the generated dynamics can be identified with the evolution of the stochastic DKCA [18]. DKCA is a model exhibiting the NEPT in the DP universality class, which is particularly simple to simulate computationally and has been used extensively to study the absorbing/percolating critical point. DKCA is a $(1+1)$-dimensional discrete stochastic CA, where the state of each cell is updated with a probability that depends on the state of its left and right neighbors. The probabilistic update rule $p\left(s_{j}^{t} \mid s_{j-1}^{t}, s_{j+1}^{t}\right)$ is given by $p(1 \mid 0,0)=x, p(1 \mid 0,1)=p(1,1,0)=y, p(1 \mid 1,1)=z$, and $p\left(0 \mid s_{j-1}^{t}, s_{j+1}^{t}\right)=1-p\left(1 \mid s_{j-1}^{t}, s_{j+1}^{t}\right)$. DKCA is block partitioned such that the updates of its even and odd cells are alternated, as shown in Fig. 1. When $x=0$, the dynamics of the DKCA correspond to DP. It is not hard to see that setting $y=z=p$ maps the dynamics to $(1+1)$-dimensional site percolation processes, where $p$ is the probability presence of a site. The model corresponds to bond percolation when
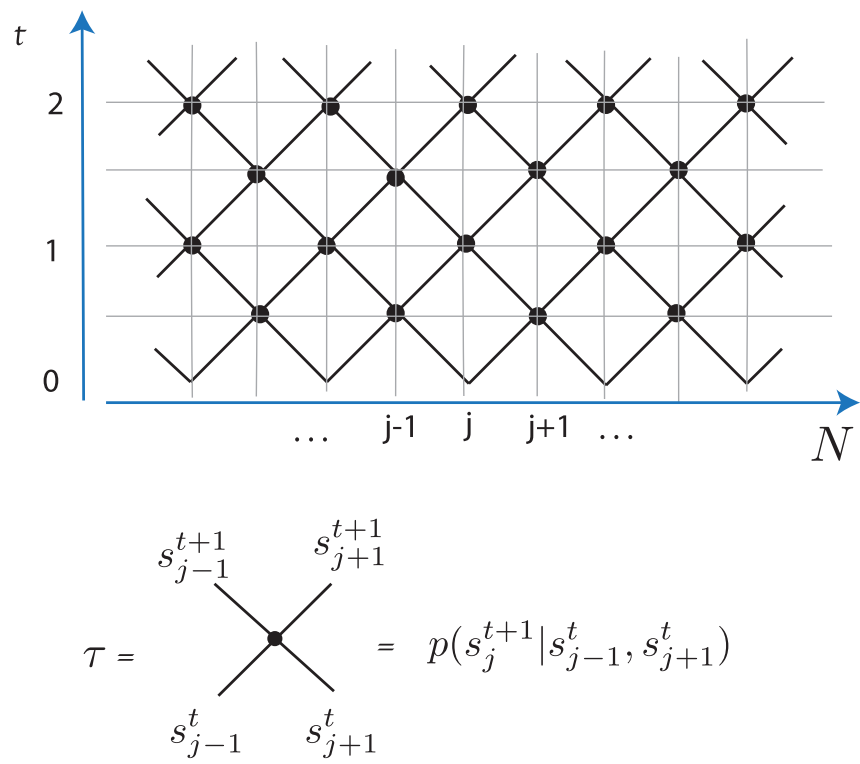

FIG. 1. A schematic representation of (1+1)-dimensional block partitioned CA with nearest-neighbor update rule, e.g., DKCA. The state of a system at time $t$ is given by contracting all the transfer matrix tensors $\tau$ in the preceding time steps.

setting $y=q$ and $z=q(2-q)$, where $q$ is the probability of presence of a bond.

For our model to correspond to DKCA stochastic rules, we will require that the site occupations in the stationary state of the CP map acting on an isolated 3-cell (a site and its neighbors) are the same as in the stationary states of the DKCA rule. Let $T$ be the transfer matrix of a DKCA 3-cell rule. Let $\mathbf{v}$ be the eigenvector of $T$ with the identity eigenvalue, $T \mathbf{v}=\mathbf{v}$. The vector $\mathbf{v}$ is the stationary state of the DKCA 3 -cell rule. The stationary state of the CP map generated by the master equation satisfies $e^{-\mathcal{L} t}\left[\rho^{s}\right]=\rho^{s}$ for all $t$ or, equivalently, $\mathcal{L}\left[\rho^{s}\right]=0$. We choose parameters $\left\{\gamma_{\alpha \beta}^{ \pm}, \theta_{\alpha \beta}\right\}$ such that the diagonal elements of $\rho^{s}$ are equal to the elements $\mathbf{v}$, i.e., $\operatorname{diag}\left(\rho^{s}\right)=\mathbf{v}$. The rates $\gamma_{\alpha \beta}^{ \pm}$are then adjusted according to

$\gamma_{\alpha \beta}^{+}=\left\{\begin{array}{ll}\frac{p_{\alpha \beta}}{1-p_{\alpha \beta}} \gamma_{\alpha \beta}^{-} & \text {if } \theta_{\alpha \beta}=0 \\ \frac{\left(1-2 p_{\alpha \beta}\right) \gamma_{\alpha \beta}^{-}+\sqrt{\left(\gamma_{\alpha \beta}^{-}\right)^{2}-16 p_{\alpha \beta} \theta_{\alpha \beta}^{2}\left(1-3 p_{\alpha \beta}+2 p_{\alpha \beta}^{2}\right)}}{2\left(1-p_{\alpha \beta}\right)} & \text { if } \theta_{\alpha \beta} \neq 0,\end{array}\right.$,

whose derivation is presented in the Appendix.

Note that for the model to be physical, the jump rates have to be positive and real. This puts a restriction on the range of allowed values of $p_{\alpha \beta}$ and $\theta_{\alpha \beta}$. If we set $\gamma_{\alpha \beta}^{-}=1$, then we find that $\gamma_{\alpha \beta}^{+}$is real and positive for $p_{\alpha \beta} \geqslant \frac{1}{2} \forall \theta_{\alpha \beta}$ or for $p_{\alpha \beta}<\frac{1}{2}$ if $\theta_{\alpha \beta} \leqslant \frac{1}{16\left(1-3 p_{\alpha \beta}+2 p_{\alpha \beta}^{2}\right)}$.

Having constructed the master equation whose dynamics implement the quantum analog of the DKCA 3-cell update rule, the remaining task is to define the order in which the updates are to be carried out. We propose to split the updates into four partitions $\mathcal{P}_{1}, \mathcal{P}_{2}, \mathcal{P}_{3}$, and $\mathcal{P}_{4}$ as shown in Fig. 2. All the cells in each partition can be updated in parallel, since the 3-cell CP maps with each partition act on distinct cells with no overlaps. 


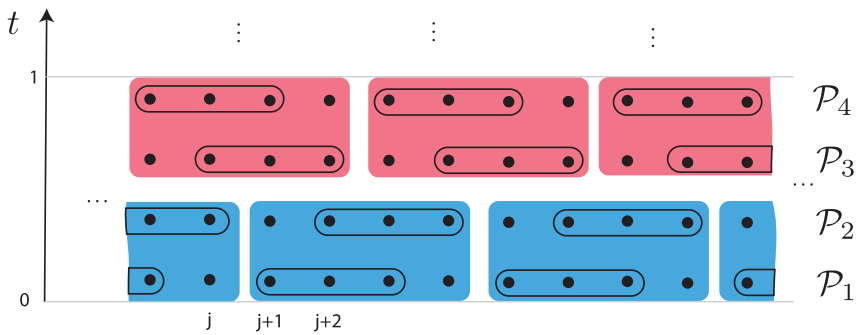

FIG. 2. Block-partitioning scheme of the proposed nonunitary QCA model of DP. Partitions $\mathcal{P}_{1}$ and $\mathcal{P}_{4}$ update even cells and partitions $\mathcal{P}_{2}$ and $\mathcal{P}_{3}$ update odd cells.

\section{NUMERICAL METHOD AND CALCULATION SETUP}

The simulation method used in this paper is based on representing the state of the system as a matrix product state (MPS) implementing time evolution using infinite time-evolving block decimation (iTEBD). MPS methods have been demonstrated to be efficient tools for studying one-dimensional quantum systems [19]. If a system has translational invariance then the iTEBD method can be used to compute the dynamics of an effectively infinite system. Though iTEBD has mostly been used to compute the unitary dynamics of a closed quantum system, it can also be applied to open dynamics with dissipation and decoherence [20].

To apply iTEBD to Lindblad dynamics given by Eq. (1), we will vectorize the density matrix using the Choi-isomorphism $|\alpha\rangle\langle\beta|\rightarrow| \alpha\rangle \otimes|\beta\rangle$. Mapped in this way the density-matrix $\rho(t)=\sum_{i j} \rho_{i j}(t)|i\rangle\langle j|$ becomes a vector in a doubled-space $|\rho(t)\rangle=\sum_{i j} \rho_{i j}(t)|i\rangle \otimes|j\rangle$. Under this mapping the Lindblad equation (1) becomes

$$
\frac{d}{d t}|\rho(t)\rangle=\mathbb{L}|\rho(t)\rangle,
$$

where the Lindblad map $\mathbb{L}$ in doubled space is given by

$$
\mathbb{L}=\mathbb{H}+\mathbb{D},
$$

with

$$
\mathbb{H}=-i\left(H \otimes I-I \otimes H^{T}\right),
$$

and

$$
\begin{aligned}
\mathbb{D}= & \sum_{ \pm} \sum_{j}\left(L_{j}^{ \pm} \otimes\left(L_{j}^{ \pm}\right)^{*}-\frac{1}{2}\left[\left(L_{j}^{ \pm}\right)^{\dagger} L_{j}^{ \pm}\right.\right. \\
& \left.\left.\otimes I+I \otimes\left(L_{j}^{ \pm}\right)^{T}\left(L_{j}^{ \pm}\right)^{*}\right]\right) .
\end{aligned}
$$

The solution to Eq. (1) is given by $|\rho(t)\rangle=e^{\mathbb{L} t}|\rho(0)\rangle$, where $|\rho(0)\rangle$ is the initial state in the vectorized representation. Defining $\mathbb{L}_{j}$ as the Liouvillian acting on the cells in the partition $\mathcal{P}_{j}$, the full round of the discrete nonunitary QCA model updating the state at step $s$ to step $s+1$ is given by

$$
\left|\rho_{s+1}\right\rangle=e^{\mathbb{L}_{4} \tau} e^{\mathbb{L}_{3} \tau} e^{\mathbb{L}_{2} \tau} e^{\mathbb{L}_{1} \tau}\left|\rho_{s}\right\rangle,
$$

where time $\tau$ is the time for which the system continuously evolves before switching to the next partition. If $\tau$ is large relative to the characteristic time of the dissipation, i.e., $\tau \gg$ $1 / \sqrt{\gamma}$, then the three cells undergoing the dynamical evolution reach a steady state, which corresponds to the state

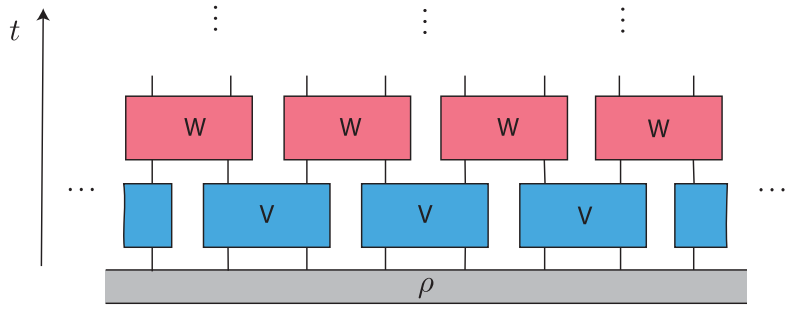

(a)

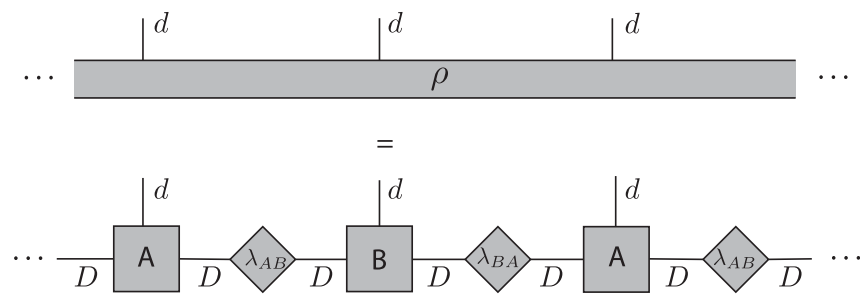

(b)

FIG. 3. (a) Tensor network representation of the single round of the QCA update with tensors $V$ and $W$ given by $V \equiv e^{\mathbb{L}_{2} \tau} e^{\mathbb{L}_{1} \tau}$ and $W=e^{\mathbb{L}_{4} \tau} e^{\mathbb{L}_{3} \tau}$. (b) MPS representation of the state vector $|\rho\rangle$. The physical index dimension is $d=16$ and the bond dimension $D$ is chosen to obtain the desired accuracy and speed of the iTEBD algorithm.

of the DKCA in the purely stochastic regime. If $\tau$ is small, i.e., $\tau \rightarrow 0$, then the partitioning can be viewed as Trotter decomposition of the time evolution $e^{\mathbb{L} t}$, where $\mathbb{L}$ acts on all sites simultaneously. Thus, in the limit $\tau \rightarrow 0$ the model can be regarded as a continuous nonunitary QCA, as the evolution happens continuously rather than in discrete steps while the notion of locality is still being preserved, as both the Hamiltonian and the Lindblad jump operators are supported over nearest neighbors. Note that although in the $\tau \gg 1 / \sqrt{\gamma}$ limit the many-body dynamics will correspond to DKCA, this is not the case for intermediate and infinitesimal values of $\tau$. In the latter case the number density in the steady state and the critical exponent may be different as will be demonstrated in Sec. IV.

Figure 3 shows the tensor network representation of the time evolution where the operator $V \equiv e^{\mathbb{L}_{2} \tau} e^{\mathbb{L}_{1} \tau}$ and $W=$ $e^{\mathbb{L}_{4} \tau} e^{\mathbb{L}_{3} \tau}$. The state $\rho$ is represented as an MPS with dimension $D$. Application of the $V$ and $W$ completely positive maps increases the bond dimension of $\rho$. In TEBD algorithms the growth of the bond dimension is controlled by only keeping a specified number of Schmidt values. From the approximate $|\rho(t)\rangle$, one can then compute the observables $\hat{O}$

$$
O(t)=\langle\hat{O}\rangle=\operatorname{tr}\left[\rho(t) \hat{O}_{D}\right]=\left\langle\mathbb{I}\left|\hat{O}_{D}\right| \rho(t)\right\rangle,
$$

where $\hat{O}_{D}=\hat{O} \otimes I$ and $|\mathbb{I}\rangle$ is the double space representation of the identity operator. 


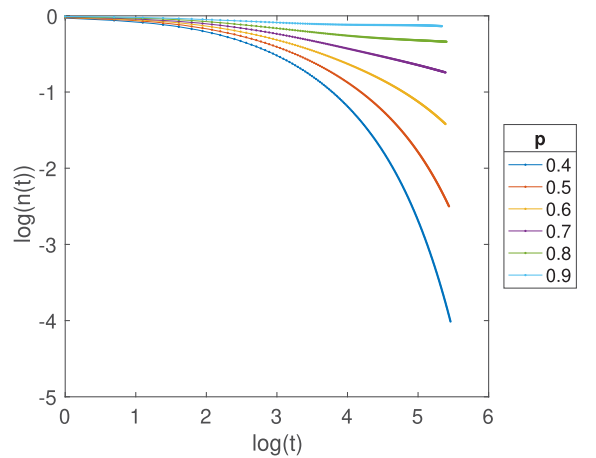

(a)

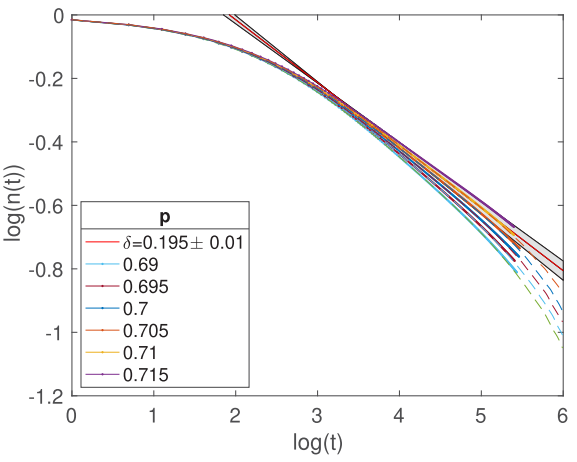

(b)

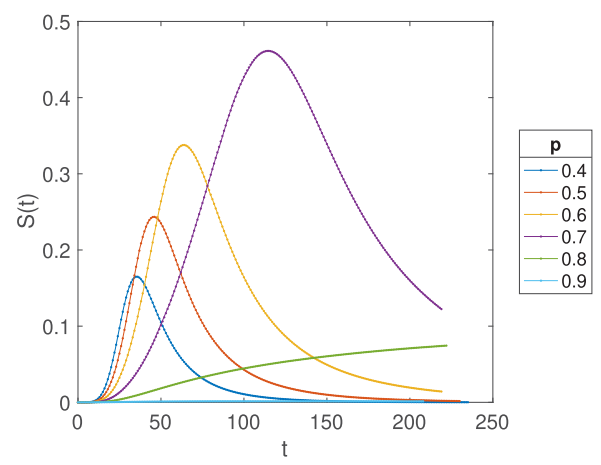

(c)

FIG. 4. Results of dynamical simulations modeling continuous time dynamics and no coherent dynamic, $\Omega=0$, using iTEBD with bond dimensions $D=128$ (dashed lines) and $D=256$ (solid lines) with a system starting in a fully occupied state. The decay rates are set to $\gamma^{+}=p$ and $\gamma^{-}=1-p$ and the duration of each partition is set to $\tau=\sqrt{0.0025 /(p(1-p))} \ll 1 / \sqrt{\gamma}$. (a) Average number density $n$ as a function of time $t$ indicating a transition between absorbing $(p \lesssim 0.7)$ and percolating ( $p \gtrsim 0.7)$ phases. (b) Average number density $n$ as a function of time $t$ for $p$ near the critical point. To determine critical exponent $\delta$, a fit to $n(t) \propto e^{-\delta t}$ for $D=128$ and 256 is carried out at critical $p_{c}=0.71$ in the time interval $t \in[35,200]$ where the deviation between higher and lower bond dimension results is less than $1 \%$ deviation. The error estimate is obtained by performing the fitting at critical and subcritical values of $p=0.71$ and $p=0.705$ with the shaded region indicating the uncertaintly in $\delta$. (c) Entropy $S(t)$ as a function of time.

\section{SIMULATIONS RESULTS AND DISCUSSION}

\section{A. Purely stochastic limit}

To verify that our nonunitary QCA model indeed represents the processes of DP on a lattice, we first compute the phase diagram of the model in the purely stochastic regime where $\theta_{\alpha \beta}=0$ for all $\alpha, \beta$. The initial state is set to $\rho=$ $|1\rangle\left\langle\left. 1\right|^{\otimes^{\infty}}\right.$ i.e., the fully active state. The time evolution is computed using the iTEBD, for various decay rates $\gamma^{+}$and $\gamma^{-}$. The decay rates are set in a ratio $\gamma^{+} / \gamma^{-}=p /(1-p)$ which corresponds to the site percolation processes with a control parameter $p$. Both continuous and discrete QCA models are considered by simulating the dynamics with a 3-cell rule generated by Liouvillian evolution with short and long durations. The lowest-order Trotter error is proportional to the commutators of the jump operators $L^{ \pm}$and hence to keep this error constant for the continuous dynamic simulations across a range of $p$ values, we adjust the time steps such that $\tau^{2} p(1-p)=C$ with the constant $C$ set to 0.0025 . For discrete dynamics simulations, we set $\tau=10.0$, which is sufficient time for a 3-cell to reach a steady state before a switch to the subsequent partitioning.

From the MPS representing the state of the system at time $t$, we compute two quantities, the average occupation density $n(t)$ and half-chain von Neumann entropy $S$, the evolution of which is well understood for the DP processes [21]. The occupation density, given by $n(t)=\operatorname{tr}(\hat{n} \rho)$ with $\hat{n}=|1\rangle\langle 1|$, can be considered as an order parameter for the transition between the absorbing and percolating phases since in the absorbing phase it is zero whereas in percolating phase it is finite. The half-chain entropy is given by $S=\left(S_{1}+\right.$ $\left.S_{2}\right) / 2$ with $S_{1,2}=-\operatorname{tr}\left[\rho_{1,2} \log \rho_{1,2}\right]$, where $\rho_{1}$ and $\rho_{2}$ are reduced density matrices of half-chain obtained by partitioning at A-B and B-A MPS bonds, respectively. The entropy is computed by first putting the MPS into the canonical form and then using equations $S_{1}=-\sum_{j}^{D} \lambda_{A B j}^{\prime 2} \log \lambda_{A B j}^{\prime 2}$ and $S_{2}=$ $-\sum_{j}^{D} \lambda_{B A j}^{\prime 2} \log \lambda_{B A j}^{\prime 2}$, where $\lambda_{A B j}^{\prime}=\lambda_{A B j} / \sum \lambda_{A B j}^{2}$ and $\lambda_{B A j}^{\prime}=$
$\lambda_{B A j} / \sum \lambda_{B A}{ }_{j}^{2}$. The evolution of the half-chain entropy is qualitatively different in the absorbing and percolating regimes. The entropy is zero in the absorbing steady state since the absorbing state is a separable product state $\rho=|0\rangle\left\langle\left. 0\right|^{\otimes^{\infty}}\right.$, while in the percolating phase, the entropy of the steady state is finite reflecting the presence of classical correlations.

Figure 4 shows the results of the simulations for the continuous QCA model. The results indicate that there is a qualitative change in evolution at $p \sim 0.7$. In the long-time limit the occupation density tends to zero for $p \lesssim 0.7$ and to a finite value for $p \gtrsim 0.7$ as can be seen in Fig. 4(a). The QCA evolution is evaluated for a finite number of time steps and the simulation does not always reach a steady state in the simulated time, particularly for values of $p$ close to the critical point where the equilibration time is very long. Nevertheless, the transition between absorbing and percolating phase is still clearly detected by evaluating the sign of the curvature of $n(t)$ on a $\log$ - $\log$ scale, i.e., for large $t, d \log n / d t<0$ in the absorbing phase, $d \log n / d t>0$ in the percolating phase, and $d \log n / d t=0$ at criticality. In Fig. 4(c), one can see that in the absorbing phase the entropy $S$ initially increases as the correlations are built up in the system but then falls off to zero as the system approaches the absorbing steady state. The entropy increases monotonically from zero to a finite value in the percolating phase.

Many nonequilibrium models, including DP, can be described using the phenomenological scaling theory. At the critical point, macroscopic observables, such as correlation length and occupation density, are determined by the power scaling laws with characteristic critical exponents. To determine how close the dynamics of our QCA model is to the DP universality class, we have computed the dynamic critical exponent $\delta$, which characterized the critical slowing down of $n(t)$ i.e., $n(t) \propto t^{-\delta}$ at $p=p_{c}$. The exponent $\delta$ is computed by first locating the critical point and then doing a linear fit of the $\log n(t)$ vs $\log t$ curve at long-time $t$ where the slope of the curve is linear. The critical point is located by 


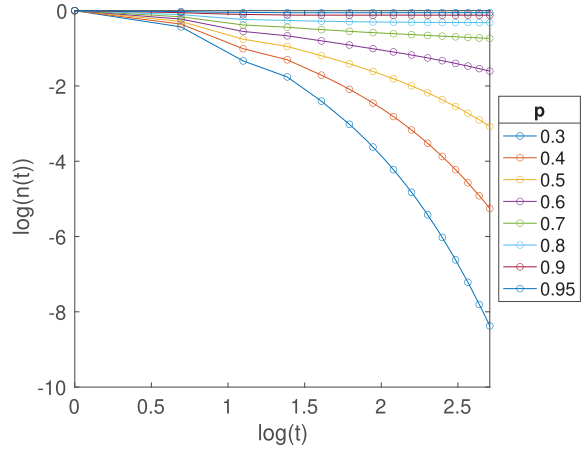

(a)

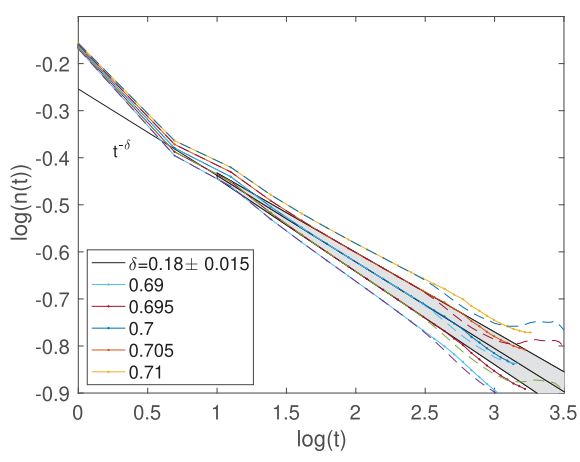

(b)

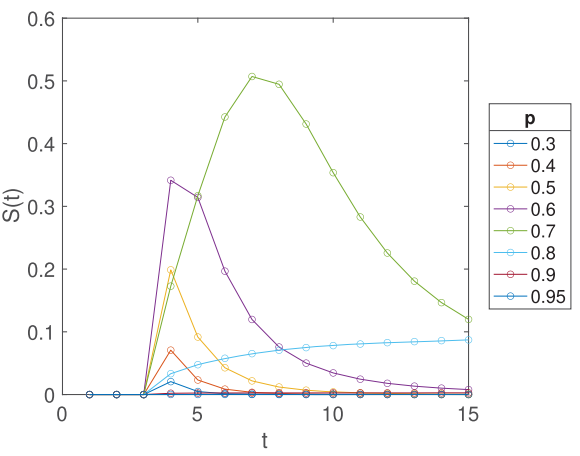

(c)

FIG. 5. Results of dynamical simulations modeling discrete dynamics and no coherent dynamic, $\Omega=0$, using iTEBD with bond dimensions $D=250$ (dashed lines) and $D=500$ (solid lines) with system starting in a fully occupied state. The decay rates are set to $\gamma^{+}=p /(1-p)$ and $\gamma^{-}=1$ and the duration of each partition is set to $\tau=10 \gg 1 / \sqrt{\gamma}$. (a) Average number density $n$ as a function of time $t$ indicating a transition between absorbing ( $p \lesssim 0.7$ ) and percolating ( $p \gtrsim 0.7$ ) phases. (b) Average number density $n$ as a function of time $t$ for $p$ near the critical point. To determine critical exponent $\delta$, a fit to $n(t) \propto e^{-\delta t}$ for $D=250$ and 500 is carried out at critical $p_{c}=0.70$ in the time interval $t \in[5,12]$ where the deviation between higher and lower bond dimension results is less than $1 \%$ deviation. The error estimate is obtained by performing the fitting at subcritical and supercritical values of $p=0.695$ and $p=0.705$ with the shaded region indicating the uncertainty in $\delta$. (c) Entropy $S(t)$ as a function of time.

scanning over a range of $p$ values and finding $p$ for which the power law scaling holds over the longest time interval. The set of $n(t)$ curves in the vicinity of the critical point for continuous dynamics is shown in Fig. 4(b). From this simulation data, we estimate $p_{c}=0.71$ and $\delta=0.195 \pm 0.01$. These values are comparable to the known values for the DKCA in the site percolation regime which are $p_{c}^{D P}=0.705$ and $\delta^{D P}=0.16[8]$. The truncation of the bond dimensions in iTEBD algorithm leads to simulation errors which build up over time. By comparing the results at a higher bond dimension $D=256$ and lower bond dimension $D=128$, we find that $n(t)$ is systematically lower. The truncation of the bond dimension at the critical point effectively reduces $n(t)$ since the contribution of the absorbing state is made larger (as it is a product state with bond dimension one). For the determination of the critical exponent $\delta$, we use the time range over which the fit using high and low bond dimension results in the deviation of less that $1 \%$. The estimate of uncertainty in $\delta$ is obtained from the available data at $p$ just above and just below critical $p_{c}$.

The results of the simulations of the dynamics in the discrete QCA limit of large $\tau$ are shown in Fig. 5. The evolution of the average occupation density in the discrete model has the same dynamical features as the continuous model, namely, $n(t)$ decays to zero for $p<p_{c}$ and to a finite value for $p>p_{c}$ with $p_{c} \sim 0.7$. The half-chain entropy also has a characteristic rising and falling behavior in the absorbing phase and monotonically rising behavior in the percolating phase as shown in Fig. 5(c). Since the Liouvillian acts for a longer time during each update step, it takes fewer steps to reach steady state and the state update is clearly discrete and discontinuous. We find that a higher bond dimension needs to be used for discrete model iTEBD simulation in order to achieve the same simulation accuracy in the continuous case. For the discrete model, we find that $p_{c}=0.70$ and the $\delta=0.18 \pm 0.015$.

In summary, we find that, remarkably, the discrete and continuous QCA models share similar collective dynamics near the critical point with $p_{c}=0.71, \delta=0.195 \pm 0.01$ (con- tinuous model) and $p_{c}=0.70, \delta=0.18 \pm 0.015$ (discrete model).

\section{B. Adding the Hamiltonian}

Having established that our nonunitary QCA model indeed corresponds to DP on a lattice, we now consider adding a local Hamiltonian term to the Liouvillian that generates the dynamical rule. The Hamiltonian can produce coherences and entanglement in the system and, therefore, by turning it on, one can systematically explore the influence of quantum effects on the DP NEPT. A Hamiltonian term of the form $|0\rangle\langle 0|\otimes X \otimes| 0\rangle\langle 0|$ is not compatible with the DK model of DP since the rate $\gamma_{00}$ given by Eq. (5) cannot be made positive with $p_{0,0}=0$. The local Hamiltonian term, $I \otimes X \otimes I$, is also incompatible with DP since its basis decomposition includes term $|0\rangle\langle 0|\otimes X \otimes| 0\rangle\langle 0|$. For this reason, we will explore the effect of the Hamiltonian, which acts on a central qubit conditioned on the neighbors being in state $|1\rangle$. The Hamiltonian, $H$, is given by

$$
H=\Omega|1\rangle\langle 1|\otimes X \otimes| 1\rangle\langle 1|,
$$

which corresponds to Eq. (2) with $\theta_{11} / 2=\Omega$ and all other $\theta_{\alpha \beta}$ are set to zero.

The jump rate constants are set to $\gamma_{00}^{-}=1, \gamma_{00}^{+}=0, \gamma_{01}^{-}=$ $\gamma_{10}^{-}=1-y, \quad \gamma_{01}^{+}=\gamma_{10}^{+}=y$, and $\gamma_{11}^{-}=1-z$, and $\gamma_{11}^{+}=$ $\frac{(2 z-1) \gamma_{11}^{-}+\sqrt{\left(\gamma_{11}^{-}\right)^{2}-16 \Omega^{2}\left(1-3 z+2 z^{2}\right)}}{2(1-z)}$. With these rates, the site occupations of the stationary state of the 3-cell rule are identical to the classical DKCA of site DP, where $x=0, y=z=p$.

The results of the iTEBD simulation for the model in the small $\tau$ limit with the Hamiltonian, $\Omega=0.1$, and no Hamiltonian, $\Omega=0$, for various values of $p$ are shown in Fig. 6 . As can be seen from Figs. 6(a) and 6(b), the evolution of the number densities and the half-chain entropy in the presence of the Hamiltonian is nearly identical with small differences of the order of $10^{-3}$. The effect of the Hamiltonian dynamics is, however, manifested much more evidently by the presence 


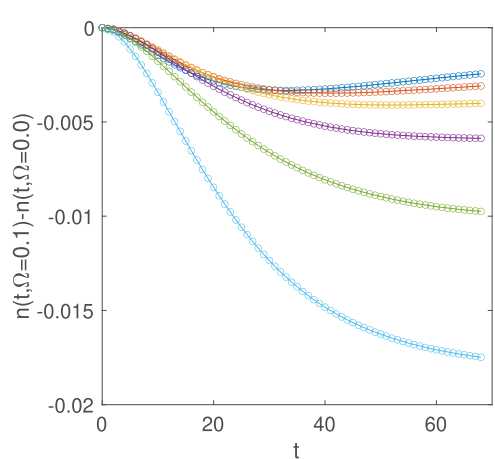

(a)

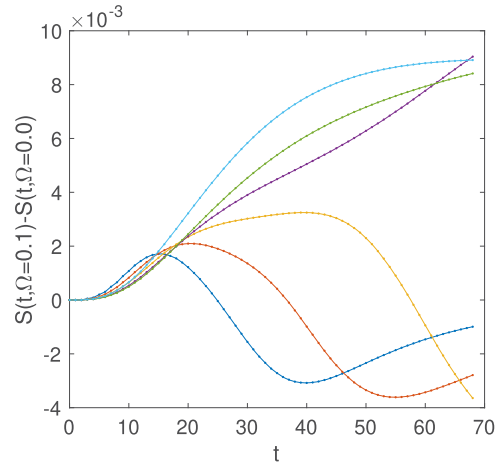

(b)
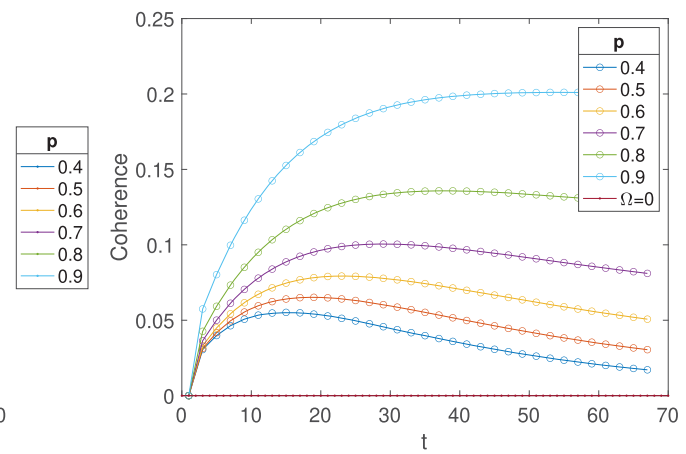

(c)

FIG. 6. Comparison of the evolution of (a) number density, (b) entropy, and (c) single-site coherence, $\left|\rho_{01}^{s}\right|+\left|\rho_{10}^{s}\right|$, for different $p$ in the presence $(\Omega=0.1)$ and absence $(\Omega=0)$ of the Hamiltonian term. The results are for $\tau=\sqrt{0.0025 /(p(1-p))}$; the curves $n(t), S(t)$ for $\Omega=0$ are shown in Fig. 4 .

of coherences in the state of the system. As a measure of coherence in the system, we will use the $\ell_{1}$ norm of coherence, given as $C_{1}(\rho)=\min _{\sigma \in \mathcal{I}}\|\rho-\sigma\|_{\ell_{1}}=\sum_{j \neq k}\left|\rho_{j, k}\right|$, where $\mathcal{I}$ is the set of all incoherent states and $\|\cdot\|_{\ell_{1}}$ is the $\ell_{1}$ matrix norm [22]. Figure 6(c) shows the time evolution of the singlesite coherence $C_{1}$ for several values of $p$. As expected, for $\Omega=0$ the coherence is always zero. For $\Omega=0.1$, the coherence is finite during the evolution and its dynamics depend on whether the system is in absorbing, $p<0.7$, or the percolating phase, $p>0.7$. For $p<0.7$, the coherence rises initially and then decays toward zero. This is because the Hamiltonian does not act on the absorbing state $\rho=|0\rangle\left\langle\left. 0\right|^{\otimes^{\infty}}\right.$ and, as the system approaches the absorbing state, the dissipation causes the coherences to gradually decay. For $p>0.7$, the coherence rises and remains finite in a percolating steady state. In the percolating steady state, the rate of generation of coherence and its rate of decay are dynamically balanced.

Apart from the coherence, an important quantum feature in a many-body dynamical system is entanglement. We have investigated the possibility of entanglement generation by the Hamiltonian given by Eq. (12) by computing concurrence $[23,24]$ in a reduced density matrix of the two adjacent sites, which is a good measure of entanglement for mixed quantum states. We found that the application of a single nonunitary DK QCA 3-cell rule on a product state $|1\rangle\langle 1|\otimes| 1\rangle\langle 1| \otimes$ $|1\rangle\langle 1|$ results in a state with nonzero concurrence. However, the concurrence disappears after applying the gates in sequence over the four partitions $\mathcal{P}_{1}, \mathcal{P}_{2}, \mathcal{P}_{3}$, and $\mathcal{P}_{4}$, and there is no many-body entanglement present in the steady state in our nonunitary QCA model.

\section{PHYSICAL IMPLEMENTATION}

The nonunitary QCA model can be effectively realized experimentally in a one dimensional array of Rydberg atoms or ensembles. In particular, to implement our nonunitary QCA model of DP one can adapt the setup [17]. In Ref. [17] each site of the one-dimensional array is a 3-level system consisting of a ground-state $|g\rangle$, a strongly interacting Rydberg state $|r\rangle$, and an excited state $|e\rangle$. The sites are equidistant and the interactions are restricted to nearest neighbors. Two laser fields couple the $|g\rangle \leftrightarrow|r\rangle$ and the $|r\rangle \leftrightarrow|e\rangle$ transitions.
The states $|g\rangle$ and $|r\rangle$ will correspond, respectively, to the empty and active states of the DP model. The energy of the Rydberg state depends on the state of the nearest neighbors and the energy level $|r\rangle$ is split into three sublevels, $\left|r_{00}\right\rangle,\left|r_{10}\right\rangle$, and $\left|r_{11}\right\rangle$, corresponding to the state when the neighboring sites are in states $|g\rangle|g\rangle,|r\rangle|g\rangle$, and $|r\rangle|r\rangle$. A multifrequency laser field couples each of the $|g\rangle \leftrightarrow|r\rangle$ and $|e\rangle \leftrightarrow|r\rangle$ transitions with tunable coupling strengths. The short-lived $|e\rangle$ decays to $|g\rangle$ with rate $\Gamma$ and is used to implement Lindblad jump operators, which depend on the state of the neighbors. After adiabatically eliminating state $|e\rangle$ the effective master equation is defined by the Hamiltonian of the form of Eq. (2) and the Lindblad operators are of the form of Eq. (4), except there are no $L^{+}$jump operators as there are no spontaneous excitation processes in the system. The missing jump operators, $L_{j}^{+}=\sum_{\alpha \beta} \sqrt{\gamma_{\alpha \beta}^{+}}|\alpha\rangle\left\langle\left.\alpha\right|_{j-1} \otimes \sigma_{j}^{+} \otimes \mid \beta\right\rangle\left\langle\left.\beta\right|_{j+1}\right.$, are needed to implement the incoherent excitation of the site $j$ conditional on the states of the neighbors. Since $\sigma^{+}=X \sigma^{-} X$, the $L^{+}$jump operators can be obtained by conjugating the system with the spin flip operators and then adjusting $\gamma_{\alpha \beta}^{ \pm}$ such that they correspond to the desired jump rates. If the time between the application of the flip pulses is small, then the action of $L^{+}$and $L^{-}$operators in the master equation can be considered to be simultaneous by the Trotterization argument.

A Rydberg atom implementation of the QCA will necessarily involve a finite number of sites. In finite-size systems there is no true absorbing/percolating phase transition because the system will always evolve toward an absorbing state as $t \rightarrow$ $\infty$. Nevertheless, one can still see the characteristics of the DP on the time scales that are long compared with the duration of a single QCA step.

\section{CONCLUSIONS}

In this paper, we have constructed a one-dimensional lattice model with local unitary and nonunitary interactions, which generalize the classical nonequilibrium process of DP into the quantum domain. The model can be viewed as a nonunitary Quantum CA with a tunable relative strength of the coherent and incoherent dissipative dynamics. The 3-cell 
rule of such nonunitary QCA is the $\mathrm{CP}$ map generated by the open nonunitary quantum dynamics specified by the choice of the local Lindblad jump operators and the Hamiltonian. Varying the duration $\tau$ of the nonunitary evolution at each time step of the QCA changes the 3-cell CP rule. We have considered the dynamics of the model in the large $\tau$ limit, which corresponds to discrete block-partitioned nonunitary QCA, and the small $\tau$ limit, which corresponds to continuous nonunitary QCA. The dynamics of the model in the two limits with no Hamiltonian term was simulated using MPS iTEBD method, and it was found that in both cases the system can undergo absorbing/percolating NEPTs. The transition point was found to be at $p=0.70$ and $p=0.71$ for the large and small $\tau$ limits, respectively, which is within $0.7 \%$ of the known critical value of $p$ for the site DP in $(1+1)$ dimensions. The dynamical exponents $\delta$ were found to be $0.18 \pm 0.015$ and $0.195 \pm 0.01$ for the discrete dynamics and the continuous dynamics model, respectively. The discrete nonunitary QCA has been constructed such that the rules correspond to DKCA if the input states are classical and thus the deviations in $p_{c}$ and $\delta$ from the classical site DP values ( $p_{c}=0.705$ and $\delta=0.16$ ) are likely due to errors in the simulation associated with the finite bond dimension, finite time, and the determination of the critical point. The continuous time model shares the same qualitative features of the DKCA, such as distinct phases characterized by local density order parameter and a similar dynamics of the half-chain entropy. Moreover, the agreement between the values of $p_{c}$ and $\delta$ in the continuous and discrete dynamics model indicates that the continuous time model is in the DP universality class.

When adding a local 3-site Hamiltonian term, the dissipation rates can be adjusted such that the evolution of the local site populations is indistinguishable from the purely dissipative dynamics. Nevertheless, such dynamics generates quantum coherences in the system as witnessed by the nonzero off-diagonal elements of the density matrix. No en- tanglement was generated by the chosen Hamiltonian and thus, an interesting open question would be to determine whether certain choices of Hamiltonians and Lindblad jump operators would generate nonunitary QCA rules that produce entangled steady states.

The proposed nonunitary QCA models can be engineered in ultracold atom experiments. We have suggested a possible way of designing the rules in the Rydberg atomic array based on the proposal in Ref. [17]. The model in the limit of small $\tau$ is equivalent to the continuous nonunitary QCA and thus can be implemented by acting on all sites with identical laser fields. This global control feature makes the scheme attractive for experimental implementation.

Our work highlights once again the potential for quantum CA for investigating emergent behavior in physical systems [25]. Since Rydberg arrays can be configured into twodimensional lattice configurations [1], a natural extension of the present work is to consider $(2+1)$-dimensional nonunitary QCA. While the generalization of the construction of the nonunitary QCA rules from one to two dimensions is straightforward, the dynamics of the two-dimensional CA is usually significantly more complex [26]. The quantum mechanical generalization of two-dimensional CA models is challenging to simulate on classical computers and for this reason their exploration is likely to be driven by experiments. Such models would constitute an excellent test bed for existing and novel tensor network algorithms.

\section{ACKNOWLEDGMENTS}

This work was supported by the Australian Research Council Centre of Excellence for Engineered Quantum Systems (Grant No. CE 170100009). This research was undertaken with the assistance of resources from the National Computational Infrastructure (NCI), which is supported by the Australian Government.

\section{APPENDIX: DERIVATION OF RATE CONSTANTS $\gamma_{\alpha \beta}^{ \pm}$}

We present a derivation of the continuous-time version of the DKCA dynamics using the Lindblad formalism. The principal idea consists of calculating the stationary states after each time step and setting them in such a way that they obey the dynamics of the desired discrete system. More specifically, the neighborhood-dependent amplitudes of the Hamiltonian $\left(\theta_{\alpha \beta}\right)$ and jump operators $\left(\gamma_{\alpha \beta}^{ \pm}\right)$are derived in accordance with the update probabilities $\left(p_{\alpha \beta}\right)$ from the DKCA.

The stationary states are determined by

$$
\mathbb{L}\left|\rho^{s}\right\rangle=0,
$$

with $\mathbb{L}$ being the vectorized version of the Liouvillian in Eqs. (7) to (9), writing

$$
\begin{aligned}
\mathbb{L}= & \sum_{\alpha \beta \alpha^{\prime} \beta^{\prime}}|\alpha\rangle\left\langle\left.\alpha\right|_{j-1} \otimes \mid \alpha^{\prime}\right\rangle\left\langle\left.\alpha^{\prime}\right|_{j^{\prime}-1}\right. \\
& \times \otimes\left[-i \frac{\theta_{\alpha \beta}}{2}\left(|0\rangle\left\langle\left. 1\right|_{j} \otimes \mid 0\right\rangle\left\langle\left. 0\right|_{j^{\prime}}+\mid 0\right\rangle\left\langle\left. 1\right|_{j} \otimes \mid 1\right\rangle\left\langle\left. 1\right|_{j^{\prime}}+\mid 1\right\rangle\left\langle\left. 0\right|_{j} \otimes \mid 0\right\rangle\left\langle\left. 0\right|_{j^{\prime}}+\mid 1\right\rangle\left\langle\left. 0\right|_{j} \otimes \mid 1\right\rangle\left\langle\left. 1\right|_{j^{\prime}}\right)\right.\right. \\
& +i \frac{\theta_{\alpha^{\prime} \beta^{\prime}}}{2}\left(|0\rangle\left\langle\left. 0\right|_{j} \otimes \mid 0\right\rangle\left\langle\left. 1\right|_{j^{\prime}}+\mid 1\right\rangle\left\langle\left. 1\right|_{j} \otimes \mid 0\right\rangle\left\langle\left. 1\right|_{j^{\prime}}+\mid 0\right\rangle\left\langle\left. 0\right|_{j} \otimes \mid 1\right\rangle\left\langle\left. 0\right|_{j^{\prime}}+\mid 1\right\rangle\left\langle\left. 1\right|_{j} \otimes \mid 1\right\rangle\left\langle\left. 0\right|_{j^{\prime}}\right)\right. \\
& +\sqrt{\gamma_{\alpha \beta}^{+} \gamma_{\alpha^{\prime} \beta^{\prime}}^{+}}|0\rangle\left\langle\left. 1\right|_{j} \otimes \mid 0\right\rangle\left\langle\left. 1\right|_{j^{\prime}}+\sqrt{\gamma_{\alpha \beta}^{-} \gamma_{\alpha^{\prime} \beta^{\prime}}^{-}} \mid 1\right\rangle\left\langle\left. 0\right|_{j} \otimes \mid 1\right\rangle\left\langle\left. 0\right|_{j^{\prime}}\right. \\
& -\frac{\gamma_{\alpha \beta}^{-}+\gamma_{\alpha^{\prime} \beta^{\prime}}^{-}}{2}|0\rangle\left\langle\left. 0\right|_{j} \otimes \mid 0\right\rangle\left\langle\left. 0\right|_{j^{\prime}}-\frac{\gamma_{\alpha \beta}^{-}+\gamma_{\alpha^{\prime} \beta^{\prime}}^{+}}{2} \mid 0\right\rangle\left\langle\left. 0\right|_{j} \otimes \mid 1\right\rangle\left\langle\left. 1\right|_{j^{\prime}}\right.
\end{aligned}
$$




$$
\begin{aligned}
& -\frac{\gamma_{\alpha \beta}^{+}+\gamma_{\alpha^{\prime} \beta^{\prime}}^{-}}{2}|1\rangle\left\langle\left. 1\right|_{j} \otimes \mid 0\right\rangle\left\langle\left. 0\right|_{j^{\prime}}-\frac{\gamma_{\alpha \beta}^{+}+\gamma_{\alpha^{\prime} \beta^{\prime}}^{+}}{2} \mid 1\right\rangle\left\langle\left. 1\right|_{j} \otimes \mid 1\right\rangle\left\langle\left. 1\right|_{j^{\prime}}\right] \\
& \times \otimes|\beta\rangle\left\langle\left.\beta\right|_{j-1} \otimes \mid \beta^{\prime}\right\rangle\left\langle\left.\beta^{\prime}\right|_{j^{\prime}-1}\right.
\end{aligned}
$$

by considering the Hamiltonian (2) and jump operators (4) $\forall \alpha, \alpha^{\prime}, \beta, \beta^{\prime} \in\{0,1\}$, where the indices $j^{\prime}$ denote the position in the dual space arising from the vectorization approach. The steady states of the system are then defined by the system of equations

$$
\begin{aligned}
\text { I. } \quad 0=\frac{-i}{2}\left(\theta_{\alpha \beta} \rho_{\alpha, \alpha^{\prime}, 1,0, \beta, \beta^{\prime}}^{s}-\theta_{\alpha^{\prime} \beta^{\prime}} \rho_{\alpha, \alpha^{\prime}, 0,1, \beta, \beta^{\prime}}^{s}\right)-\frac{\gamma_{\alpha \beta}^{-}+\gamma_{\alpha^{\prime} \beta^{\prime}}^{-}}{2} \rho_{\alpha, \alpha^{\prime}, 0,0, \beta, \beta^{\prime}}^{s}+\sqrt{\gamma_{\alpha \beta}^{+} \gamma_{\alpha^{\prime} \beta^{\prime}}^{+}} \rho_{\alpha, \alpha^{\prime}, 1,1, \beta, \beta^{\prime}}^{s} \\
\text { II. } \quad 0=\frac{-i}{2}\left(\theta_{\alpha \beta} \rho_{\alpha, \alpha^{\prime}, 1,1, \beta, \beta^{\prime}}^{s}-\theta_{\alpha^{\prime} \beta^{\prime}} \rho_{\alpha, \alpha^{\prime}, 0,0, \beta, \beta^{\prime}}^{s}\right)-\frac{\gamma_{\alpha \beta}^{-}+\gamma_{\alpha^{\prime} \beta^{\prime}}^{+}}{2} \rho_{\alpha, \alpha^{\prime}, 0,1, \beta, \beta^{\prime}} \\
\text { III. } \quad 0=\frac{-i}{2}\left(\theta_{\alpha \beta} \rho_{\alpha, \alpha^{\prime}, 0,0, \beta, \beta^{\prime}}-\theta_{\alpha^{\prime} \beta^{\prime}} \rho_{\alpha, \alpha^{\prime}, 1,1, \beta, \beta^{\prime}}\right)-\frac{\gamma_{\alpha \beta}^{+}+\gamma_{\alpha^{\prime} \beta^{\prime}}^{-}}{2} \rho_{\alpha, \alpha^{\prime}, 1,0, \beta, \beta^{\prime}}^{s} \\
\text { IV. } \quad 0=\frac{-i}{2}\left(\theta_{\alpha \beta} \rho_{\alpha, \alpha^{\prime}, 0,1, \beta, \beta^{\prime}}^{s}-\theta_{\alpha^{\prime} \beta^{\prime}} \rho_{\alpha, \alpha^{\prime}, 1,0, \beta, \beta^{\prime}}^{s}\right)-\frac{\gamma_{\alpha \beta}^{+}+\gamma_{\alpha^{\prime} \beta^{\prime}}^{+}}{2} \rho_{\alpha, \alpha^{\prime}, 1,1, \beta, \beta^{\prime}}^{s}+\sqrt{\gamma_{\alpha \beta}^{-} \gamma_{\alpha^{\prime} \beta^{\prime}}^{-}} \rho_{\alpha, \alpha^{\prime}, 0,0, \beta, \beta^{\prime}}^{s},
\end{aligned}
$$

where the first and last two subscripts of the steady-state elements $\rho_{\alpha, \alpha^{\prime}, c, c^{\prime}, \beta, \beta^{\prime}}^{s}$ denote the basis of the left or right sites, ( $j-$ $\left.1, j^{\prime}-1\right)$ or $\left(j+1, j^{\prime}+1\right)$, respectively, while the center site $\left(j, j^{\prime}\right)$ is associated with $c, c^{\prime} \in\{0,1\}$.

Without loss of generality one can consider that the nearest-neighbor states and their dual space correspondence are identical, setting $\alpha=\alpha^{\prime}$ and $\beta=\beta^{\prime}$. The Liouvillian is then simplified to

$$
\begin{aligned}
\mathbb{L}= & \sum_{\alpha \beta}|\alpha\rangle\left\langle\left.\alpha\right|_{j-1} \otimes \mid \alpha\right\rangle\left\langle\left.\alpha\right|_{j^{\prime}-1}\right. \\
& \times \otimes\left[\frac { - i \theta _ { \alpha \beta } } { 2 } \left(| 0 \rangle \langle 1 | _ { j } \otimes | 0 \rangle \langle 0 | _ { j ^ { \prime } } + | 0 \rangle \langle 1 | _ { j } \otimes | 1 \rangle \langle 1 | _ { j ^ { \prime } } + | 1 \rangle \langle 0 | _ { j } \otimes | 0 \rangle \langle 0 | _ { j ^ { \prime } } + | 1 \rangle \langle 0 | _ { j } \otimes | 1 \rangle \left\langle\left.1\right|_{j^{\prime}}\right.\right.\right. \\
& -|0\rangle\left\langle\left. 0\right|_{j} \otimes \mid 0\right\rangle\left\langle\left. 1\right|_{j^{\prime}}-\mid 1\right\rangle\left\langle\left. 1\right|_{j} \otimes \mid 0\right\rangle\left\langle\left. 1\right|_{j^{\prime}}-\mid 0\right\rangle\left\langle\left. 0\right|_{j} \otimes \mid 1\right\rangle\left\langle\left. 0\right|_{j^{\prime}}-\mid 1\right\rangle\left\langle\left. 1\right|_{j} \otimes \mid 1\right\rangle\left\langle\left. 0\right|_{j^{\prime}}\right) \\
& +\gamma_{\alpha \beta}^{+}\left(|0\rangle\left\langle\left. 1\right|_{j} \otimes \mid 0\right\rangle\left\langle\left. 1\right|_{j^{\prime}}-\mid 1\right\rangle\left\langle\left. 1\right|_{j} \otimes \mid 1\right\rangle\left\langle\left. 1\right|_{j^{\prime}}\right)\right. \\
& +\gamma_{\alpha \beta}^{-}\left(|1\rangle\left\langle\left. 0\right|_{j} \otimes \mid 1\right\rangle\left\langle\left. 0\right|_{j^{\prime}}-\mid 0\right\rangle\left\langle\left. 0\right|_{j} \otimes \mid 0\right\rangle\left\langle\left. 0\right|_{j^{\prime}}\right)\right. \\
& -\frac{\gamma_{\alpha \beta}^{-}+\gamma_{\alpha \beta}^{+}}{2}\left(|0\rangle\left\langle\left. 0\right|_{j} \otimes \mid 1\right\rangle\left\langle\left. 1\right|_{j^{\prime}}+\mid 1\right\rangle\left\langle\left. 1\right|_{j} \otimes \mid 0\right\rangle\left\langle\left. 0\right|_{j^{\prime}}\right)\right] \\
& \times \otimes|\beta\rangle\left\langle\left.\beta\right|_{j-1} \otimes \mid \beta\right\rangle\left\langle\left.\beta\right|_{j^{\prime}-1} .\right.
\end{aligned}
$$

For clarity the subscripts $\alpha, \beta$ are omitted further by replacing $\gamma_{\alpha \beta}^{ \pm} \rightarrow \gamma^{ \pm}, p_{\alpha \beta} \rightarrow p, \theta_{\alpha \beta} / 2 \rightarrow \Omega$ and $\rho_{\alpha, \alpha^{\prime}, c, c^{\prime}, \beta, \beta^{\prime}}^{s}=$ $\rho_{\alpha, \alpha, c, c^{\prime}, \beta, \beta}^{s} \rightarrow \rho_{c, c^{\prime}}^{s}$, yielding a simplified system of equations that define the steady-state elements $\forall \alpha, \beta$ :

$$
\begin{array}{ll}
\text { I. } & 0=i \Omega\left(\rho_{01}^{s}-\rho_{10}^{s}\right)-\gamma^{-} \rho_{00}^{s}+\gamma^{+} \rho_{11}^{s} \\
\text { II. } & 0=i \Omega\left(\rho_{00}^{s}-\rho_{11}^{s}\right)-\frac{\gamma^{+}+\gamma^{-}}{2} \rho_{01}^{s} \\
\text { III. } & 0=i \Omega\left(\rho_{11}^{s}-\rho_{00}^{s}\right)-\frac{\gamma^{+}+\gamma^{-}}{2} \rho_{10}^{s} \\
\text { IV. } & 0=i \Omega\left(\rho_{10}^{s}-\rho_{01}^{s}\right)+\gamma^{-} \rho_{00}^{s}-\gamma^{+} \rho_{11}^{s},
\end{array}
$$

where it is noted that Eq. (A5) II and III demand $\rho_{01}^{s}=\left(\rho_{10}^{s}\right)^{*}$ and Eq. (A5) I and IV lead to $\rho_{00}^{s}+\rho_{11}^{s}=1$ representing the trace-preserving property of the reduced density matrix at site $j$.

Desired dynamics are thus encoded into the parameters that describe the system $\gamma^{ \pm}$and $\Omega$. As $\Omega$ describes the strength of the Hamiltonian which tunes quantum coherences, this shall not be fixed but remain a variable to effectively increase or decrease the quantum coherences, turning them on $(\Omega \neq 0)$ or off $(\Omega=0)$.

Here the stationary states of the system are set after each time step to obey the dynamics of the DKCA. In this model an arbitrary input state is taken to $\left(\begin{array}{cc}p & 0 \\ 0 & 1-p\end{array}\right)$, i.e., the system is in the classical " 1 " state with probability $p$ or in " 0 " with probability $1-p$ after one time step. The corresponding quantum dynamics are described in the Lindblad formalism by setting $\rho_{11}^{s}=1-p$ and then solving the system of equations (17) for one of the variables that describe the system, say $\gamma^{+}$. By introducing the Hamiltonian, part of the Liouvillian quantum coherences show up as the off-diagonal matrix elements of the density matrix 
$\left(\begin{array}{cc}p & \rho_{01}^{s} \\ -\rho_{01}^{s} & 1-p\end{array}\right)$ with

$$
\rho_{01}^{s}=\left\{\begin{array}{ll}
0 & , \text { if } \Omega=0 \\
\frac{-i}{4 \Omega}\left(\gamma^{-}-\sqrt{\left(\gamma^{-}\right)^{2}-16 \Omega^{2}\left(1-3 p+2 p^{2}\right)}\right) & , \text { if } \Omega \neq 0
\end{array},\right.
$$

where

$$
\gamma^{+}=\left\{\begin{array}{ll}
\frac{p}{1-p} \gamma^{-} & \text {, if } \Omega=0 \\
\frac{(2 p-1) \gamma^{-}+\sqrt{\left(\gamma^{-}\right)^{2}-16 \Omega^{2}\left(1-3 p+2 p^{2}\right)}}{2(1-p)} & , \text { if } \Omega \neq 0
\end{array},\right.
$$

which is, e.g., with $\gamma^{-}=1$ only real and positive in the cases

$$
\begin{aligned}
& \text { I. } \Omega=0 \\
& \text { II. } \quad p \geqslant \frac{1}{2} \forall \Omega \\
& \text { III. } p<\frac{1}{2} \text { if } \Omega \leqslant \frac{1}{16\left(1-3 p+2 p^{2}\right)} .
\end{aligned}
$$

Note that for our simulations of the DP model the Hamiltonian is chosen to be only turned on if both neighboring states are in the 1 state, i.e., we set $\Omega=\theta_{11} / 2$ where $\alpha=\beta=1$.

[1] A. Browaeys and T. Lahaye, Many-body physics with individually controlled Rydberg atoms, Nat. Phys. 16, 132 (2020).

[2] H. Bernien, S. Schwartz, A. Keesling, H. Levine, A. Omran, H. Pichler, S. Choi, A. S. Zibrov, M. Endres, M. Greiner, V. Vuletić, and M. D. Lukin, Probing many-body dynamics on a 51-atom quantum simulator, Nature (London) 551, 579 (2017).

[3] E. Guardado-Sanchez, P. T. Brown, D. Mitra, T. Devakul, D. A. Huse, P. Schauß, and W. S. Bakr, Probing the Quench Dynamics of Antiferromagnetic Correlations in a 2D Quantum Ising Spin System, Phys. Rev. X 8, 021069 (2018).

[4] D. Barredo, H. Labuhn, S. Ravets, T. Lahaye, A. Browaeys, and C. S. Adams, Coherent Excitation Transfer in a Spin Chain of Three Rydberg Atoms, Phys. Rev. Lett. 114, 113002 (2015).

[5] S. de Léséleuc, V. Lienhard, P. Scholl, D. Barredo, S. Weber, N. Lang, H. P. Büchler, T. Lahaye, and A. Browaeys, Observation of a symmetry-protected topological phase of interacting bosons with Rydberg atoms, Science 365, 775 (2019).

[6] H. Kim, Y. J. Park, K. Kim, H.-S. Sim, and J. Ahn, Detailed Balance of Thermalization Dynamics in Rydberg-Atom Quantum Simulators, Phys. Rev. Lett. 120, 180502 (2018).

[7] C. J. Turner, A. A. Michailidis, D. A. Abanin, M. Serbyn, and Z. Papić, Weak ergodicity breaking from quantum many-body scars, Nat. Phys. 14, 745 (2018).

[8] H. Hinrichsen, Non-equilibrium critical phenomena and phase transitions into absorbing states, Adv. Phys. 49, 815 (2000).

[9] G. Ódor, Universality classes in nonequilibrium lattice systems, Rev. Mod. Phys. 76, 663 (2004).

[10] M. Marcuzzi, M. Buchhold, S. Diehl, and I. Lesanovsky, Absorbing State Phase Transition with Competing Quantum and Classical Fluctuations, Phys. Rev. Lett. 116, 245701 (2016).

[11] F. Carollo, E. Gillman, H. Weimer, and I. Lesanovsky, Critical Behavior of the Quantum Contact Process in One Dimension, Phys. Rev. Lett. 123, 100604 (2019).
[12] E. Gillman, F. Carollo, and I. Lesanovsky, Numerical simulation of critical dissipative non-equilibrium quantum systems with an absorbing state, New J. Phys. 21, 093064 (2019).

[13] I. Lesanovsky, K. Macieszczak, and P. J. Garrahan, Nonequilibrium absorbing state phase transitions in discretetime quantum cellular automaton dynamics on spin lattices, Quantum Sci. Technol. 4, 02LT02 (2019).

[14] E. Gillman, F. Carollo, and I. Lesanovsky, Nonequilibrium Phase Transitions in $(1+1)$-Dimensional Quantum Cellular Automata with Controllable Quantum Correlations, Phys. Rev. Lett. 125, 100403 (2020).

[15] E. Gillman, F. Carollo, and I. Lesanovsky, Numerical simulation of quantum nonequilibrium phase transitions without finite-size effects, Phys. Rev. A 103, L040201 (2021).

[16] E. Gillman, F. Carollo, and I. Lesanovsky, Quantum and classical temporal correlations in $(1+1) \mathrm{D}$ quantum cellular automata, arXiv:2104.04279.

[17] T. M. Wintermantel, Y. Wang, G. Lochead, S. Shevate, G. K. Brennen, and S. Whitlock, Unitary and Nonunitary Quantum Cellular Automata with Rydberg Arrays, Phys. Rev. Lett. 124, 070503 (2020).

[18] E. Domany and W. Kinzel, Equivalence of Cellular Automata to Ising Models and Directed Percolation, Phys. Rev. Lett. 53, 311 (1984).

[19] U. Schollwöck, The density-matrix renormalization group, Rev. Mod. Phys. 77, 259 (2005).

[20] R. Orús and G. Vidal, Infinite time-evolving block decimation algorithm beyond unitary evolution, Phys. Rev. B 78, 155117 (2008).

[21] K. Harada and N. Kawashima, Entropy Governed by the Absorbing State of Directed Percolation, Phys. Rev. Lett. 123, 090601 (2019).

[22] C. Carmeli, T. Heinosaari, S. Maniscalco, J. Schultz, and A. Toigo, Determining quantum coherence with minimal resources, New J. Phys. 20, 063038 (2018). 
[23] W. K. Wootters, Entanglement of Formation of an Arbitrary State of Two Qubits, Phys. Rev. Lett. 80, 2245 (1998).

[24] A. Wong and N. Christensen, Potential multiparticle entanglement measure, Phys. Rev. A 63, 044301 (2001).
[25] L. E. Hillberry, M. T. Jones, D. L. Vargas, P. Rall, N. Y. Halpern, N. Bao, S. Notarnicola, S. Montangero, and L. D. Carr, Entangled quantum cellular automata, physical complexity, and Goldilocks rules, Quantum Sci. Technol. 6, 045017 (2021).

[26] P. Rujàn, Cellular automata and statistical mechanical models, J. Stat. Phys. 49, 139 (1987). 